\title{
Molecular Organic Electronic Circuits
}

\author{
Vladimir Bulovi \\ Microsystems Technology \\ Laboratories \\ 77 Massachusetts Ave., RM 13-3138 \\ Cambridge, MA \\ 001-617-253-7012 \\ bulovic@mit.edu \\ Kevin Ryu \\ Microsystems Technology \\ Laboratories \\ 77 Massachusetts Ave., RM 38-265 \\ Cambridge, MA \\ 001-617-452-4333 \\ ryu@mit.edu \\ Charles G. Sodini \\ Microsystems Technology \\ Laboratories \\ Ioannis Kymissis \\ Columbia University \\ 500 W120th Street 1301 \\ New York, NY 10027 \\ 001-212-854-4023 \\ johnkym@ee.columbia.edu \\ Annie Wang \\ Microsystems Technology \\ Laboratories \\ 77 Massachusetts Ave., RM 39-655 \\ Cambridge, MA \\ 001-617-253-0722 \\ aiwang@mit.edu \\ 77 Massachusetts Ave., RM 39-527B \\ Cambridge, MA \\ 001-617-253-4938 \\ sodini@mit.edu
}

\author{
Ivan Nausieda \\ Microsystems Technology \\ Laboratories \\ 77 Massachusetts Ave., RM 13-3146 \\ Cambridge, MA \\ 001-617-452-3194
}

nausieda@mit.edu

Akintunde Ibitayo Akinwande

Microsystems Technology

Laboratories

77 Massachusetts Ave., RM 39-533

Cambridge, MA

001-617-258-7974

akinwand@mtl.mit.edu

\begin{abstract}
Electronic energy disorder associated within amorphous and polycrystaline molecular organic thin film structures strongly affects the macroscopic observable behavior of organic field effect transistors (OFET) and poses practical challenges to implementing OFET circuits. It has been convenient to ignore the detailed physical processes associated with this disorder and model OFET behavior as equivalent to silicon FETs, but such simplifications limit our ability to develop integrated circuit technology as they fail to predict the true integrated OFET behavior. This talk will highlight the evolution of the organic electronic circuit technology and the challenges that lay ahead, emphasizing the need for physically accurate models of device behavior as the cornerstone of any future circuit advancements.
\end{abstract}

Permission to make digital or hard copies of all or part of this work for personal or classroom use is granted without fee provided that copies are not made or distributed for profit or commercial advantage and that copies bear this notice and the full citation on the first page. To copy otherwise, to republish, to post on servers or to redistribute to lists, requires prior specific permission and/or a fee.

ICCAD'06, November 5-9, 2006, San Jose, CA

Copyright 2006 ACM 1-59593-389-1/06/0011 ...\$5.00

\section{Categories and Subject Descriptors \\ B.7.0 [Integrated Circuits]: General.}

\section{General Terms}

Measurement, Design, Experimentation, Standardization.

\section{Keywords}

OFET, TFT, organic transistor, characterization, integrated circuits.

\section{INTRODUCTION}

This paper argues for standardization of characterization techniques for OFETs, employing methods that differ from those utilized for Si FET analysis. In particular, we discuss the shortcomings in measuring OFET behavior with conventional $\mathrm{Si}$ methods for determining the threshold voltage $\left(\mathrm{V}_{\text {th }}\right)$, mobility, and contact resistance. We then present alternate methods for extracting these physical parameters that are needed for organic integrated circuit design

\section{Characertizing OFETs}

Interest in the organic FET technology is sustained by the promise of ubiquitous and large area electronics. The low temperature growth of organic thin film structures through thermal evaporation and solution processing, enables OFET technology to be developed on plastic substrates or flexible metal foils, as needed for large area, transparent, and flexible applications. Additionally, 
the same deposition methods are employed in the growth of organic photosensitive, light emitting, and chemosensitive devices suggesting a seamless integration of a variety of dissimilar organic electronic devices. Simple integrated systems such as arrays of organic photodetectors and pressure sensors have already been demonstrated using OFET drivers [1,2]. The next step is designing more complex systems with organic analog circuits.

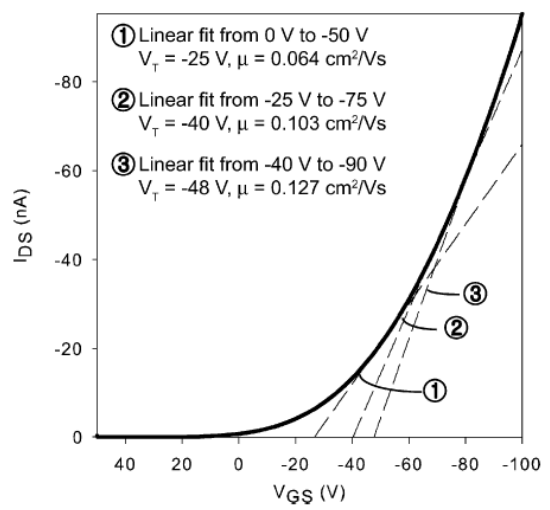

Figure 1. Extraction of mobility and threshold voltage using the silicon long channel FET model. These extracted parameters vary greatly with the range used for the linear fit. From Ref. [4]

In order to do so, it is necessary to first develop a standard method for fabricating organic integrated circuits [3]. Second, accurate OFET characterization is needed to enable us to predict OFET behavior in an integrated circuit, rather than just as isolated elements, as in the preponderance of present studies.

In 2004, the IEEE published a standard to characterize OFET behavior, assuming a Si long channel FET model for mobility and $\mathrm{V}_{\text {th }}$ extraction. Unfortunately, this model is inaccurate because it does not take into account the electronic trap distribution in the device channel, nor does it address the significant non-linear contact resistance at the source drain contacts. Fig. 1 and study of Ref. [4] highlight the shortcomings of the Si long channel model when applied to extraction of OFET charge carrier mobility. In Fig. 2 we show the ambiguity in modeling mobility in OFETs with large contact resistance, which arises from the material, energy level and charge density discontinuities at the undoped organic/metal junctions of the source/drain contacts. The carrier injection barrier at the metal/organic contact can also be temperature dependent, as can the transport through the channel of the organic semiconducting material, further highlighting the need for careful device measurement and modeling.
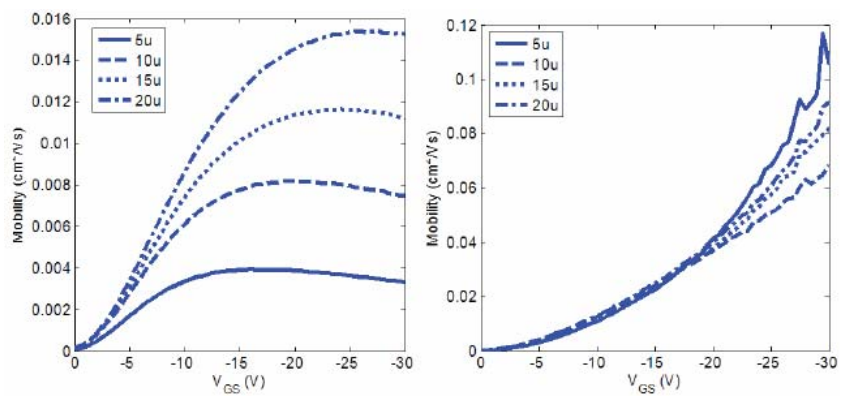

Figure 2. (left) Mobility extraction without removing contact resistance. (right) When contact resistance is accounted for, mobility increases with $V_{g s}$ as shown in [4].

Obtaining contact resistance via transmission line measurements and removing it from mobility measurements results in the physically correct dependence of mobility with gate bias [4].

\section{Conclusion}

Standard silicon device characterization techniques are shown to be insufficient in modeling the full behavior of OFETs. We show that OFET contact resistance often plays an important role in device performance and must be taken into account in device characterization in order to capture the physical behavior of charge transport in the channel.

\section{ACKNOWLEDGMENTS}

This work was supported by the MARCO C2S2 and MSD Focus Research Centers.

\section{REFERENCES}

[1] I. Kymissis, C.G. Sodini, A.I. Akinwande, V. Bulović, IEDM Tech. Digest, 377 (2004).

[2] T. Someya et al., IEEE Int'l. Solid State Circuits Conf. Dig. of Tech. Papers, 288 (2004).

[3] I. Kymissis, A.I. Akinwande, V. Bulović, IEEE J. Display Tech. 1, 289 (2005).

[4] K. Ryu, I.Kymissis, V.Bulović, C.G. Sodini, IEEE Electron Device Letters, 26, 716 (2005). 